\title{
Role of blood urea nitrogen and serum albumin ratio in predicting severity of community acquired pneumonia (CAP)
}

\author{
Mehul Agarwal $^{1}$, Madhur Joshi ${ }^{2}$, Manohar Gupta ${ }^{3}$, Neha Bharti ${ }^{4}$, Amartya Chakraborti ${ }^{1}$, Maldev Sonigra ${ }^{1}$ \\ ${ }^{1}$ Department of Pulmonary Medicine, Sleep and Critical Care, All India Institute of Medical Sciences, Jodhpur; \\ ${ }^{2}$ Rajasthan Hospital, Jaipur; ${ }^{3}$ Consultant Pulmonologist and Head, Santokba Durlabhji Memorial Hospital, Jaipur; \\ ${ }^{4}$ Department of Anaesthesiology and Critical Care, All India Institute of Medical Sciences, Jodhpur, India
}

\begin{abstract}
Blood urea nitrogen and serum albumin levels are independent risk factors for poor clinical outcome in CAP. However, there
\end{abstract}

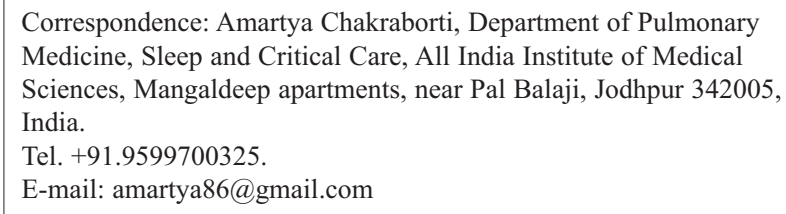

E-mail: amartya86@gmail.com

Key words: B/A ratio; community acquired pneumonia; CURB-65.

Contributions: MA, NB, MS, involved in patient enrolment, work up of patients, data entry and manuscript preparation; MG, MJ, involved in study formulation, manuscript preparation and statistical analysis; $\mathrm{AC}$, involved in study formulation, data analysis, statistical evaluation and manuscript preparation. All the authors have read and approved the final version of the manuscript and agreed to be accountable for all aspects of the work.

Conflict of interest: The authors declare that they have no competing interests, and all authors confirm accuracy.

Ethics approval and consent to participate: The study was approved by the Institute Ethical Committee.

Availability of data and material: Data can be shared on reasonable request.

Received for publication: 18 September 2021.

Accepted for publication: 22 November 2021.

Publisher's note: All claims expressed in this article are solely those of the authors and do not necessarily represent those of their affiliated organizations, or those of the publisher, the editors and the reviewers. Any product that may be evaluated in this article or claim that may be made by its manufacturer is not guaranteed or endorsed by the publisher.

${ }^{\circ}$ Copyright: the Author(s), 2021

Licensee PAGEPress, Italy

Monaldi Archives for Chest Disease 2022; 92:2091

doi: 10.4081/monaldi.2021.2091

This article is distributed under the terms of the Creative Commons Attribution-NonCommercial International License (CC BY-NC 4.0) which permits any noncommercial use, distribution, and reproduction in any medium, provided the original author(s) and source are credited. is a paucity in the literature on the role of blood urea nitrogen and albumin ratio (B/A) in CAP. This was a prospective observational study in which 112 admitted patients with the diagnosis of CAP underwent routine blood examinations, $\mathrm{ABG}$, procalcitonin and chest X-ray. Univariate analysis among various risk factors, CURB-65 scores, blood parameters including B/A ratios and clinical outcomes were carried out followed by multiple logistic regression. Cox regression was done to look at $\mathrm{B} / \mathrm{A}$ values and time to mortality. In the logistic regression, age, CURB-65 score, $\mathrm{B} / \mathrm{A}$ ratio and procalcitonin came out to be independent risk factors for ICU admission and mortality. Odds ratio of B/A in predicting mortality and ICU admission came out to be $67.8(49.2-$ 95.4) and 11.2 (8.4-14), respectively. Cox regression showed B/A values were also found to have a statistically significant relationship with time to mortality ( $\mathrm{p}=0.001$ ). $\mathrm{B} / \mathrm{A}$ ratio has the potential to become a veritable predictor of poor clinical outcomes in patients with CAP.

\section{Introduction}

Community-acquired pneumonia (CAP) is one of the leading causes of mortality and morbidity in the world. A recent systemic analysis revealed that 2.4 million deaths occur every year among all ages due to lower respiratory tract infections (LRTIs) [1]. In recent years, there has been a steady increase in the hospitalization rates including intensive care units (ICU) due to CAP, especially in the older population [2]. Scoring systems have been developed over the years to ascertain the risk factors associated with worse clinical outcomes in patient with CAP. One such extremely popular and easy to carry out test is CURB-65, advocated by the British Thoracic Society and includes parameters like presence of confusion, serum urea levels, respiratory rate, blood pressure and age of the patient [3]. An issue with this scoring system is the parameter of confusion which is quite difficult to judge, especially in aged patients with dementia and other neurological disorders and as a result the score might vary between clinicians [4,5]. In numerous studies it has been shown that blood urea nitrogen and serum albumin levels are independent risk factors for poor clinical outcome in CAP $[3,6,7]$. Hence blood urea nitrogen divided by serum albumin levels (B/A) can be used as a veritable blood marker to prognosticate CAP patients. Other than a few studies [8,9], in general, there is a paucity of literature on the role of B/A in CAP, especially from a resource constrained country like India. Hence, we carried out a prospective study aimed to look at the predictive power of $\mathrm{B} / \mathrm{A}$ ratio and also to compare it with a standard scoring system like CURB -65 in CAP. 


\section{Materials and Methods}

This was a prospective observational study in which consecutive patients with the diagnosis of CAP and requiring indoor admission from June 2019 to December 2019 were enrolled after due consent. Study was carried out in a tertiary level hospital in the Indian State of Rajasthan and the study was approved by the Institute Ethical Committee. Refusal to consent to take part in the study was the only exclusion criteria. Study period was of 6 months and the patients were followed up for up to 30 days of admission.

Following enrolment after due consent, all patients underwent a thorough clinical examination and clinical history taking. Clinical risk factors that can predict worse clinical outcomes were also tabulated. Comorbidities like respiratory ailments (including COPD, ILD, post tubercular sequelae), cardiac ailments (including ischaemic heart disease, hypertension), chronic kidney disease, diabetes mellitus were noted. Routine blood parameters like CBC, LFT, KFT, serum electrolytes, procalcitonin, sputum pyogenic and fungal culture, Ziehl Neelsen staining, mycobacterial culture and chest X-ray were done for all patients at admission. Routine ABG at time of admission and procalcitonin was done for all patients. If clinically indicated, nasopharyngeal swabs were taken and sent for H1N1 RT-PCR. Other tests like CT scan or bronchoscopy guided respiratory sample collection were carried out as per the decisions of the treating physician. All patients were followed up for a period of 30 days to look for mortality and requirement of ICU admission.

ROC curve of CURB -65 as a predictor of Mortality

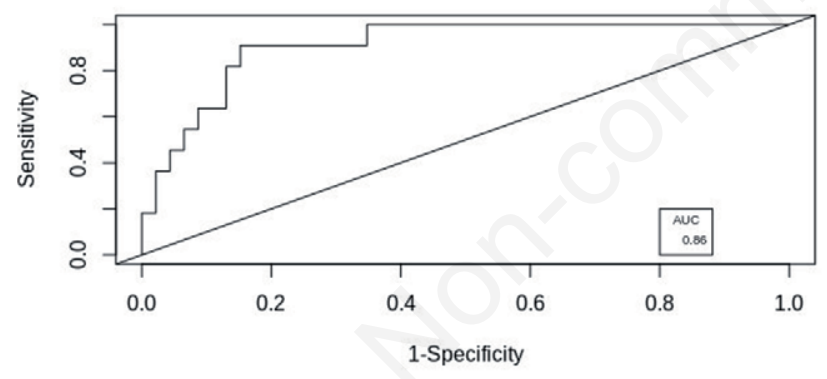

Figure 1. ROC curve of CURB-65 as a predictor of mortality.

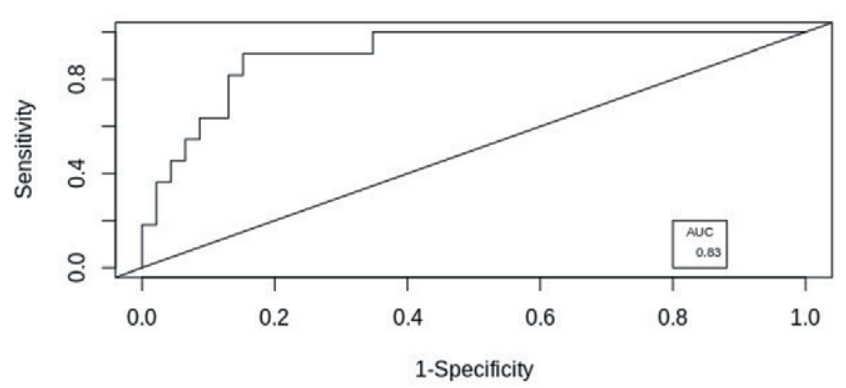

Figure 2. ROC curve of CURB-65 as a predictor of ICU admission.

\section{Statistical analysis}

Patient parameters were put into Microsoft Excel and then fed into R studio v. 1.2.5019. Univariate analysis among various risk factors and clinical outcomes were carried out followed by multiple logistic regression; p-value $<0.05$ was taken to be significant. ROC curve was done for CURB-65 scores (Figures 1 and 2) and $\mathrm{B} / \mathrm{A}$ ratios (Figures 3 and 4 ) with respect to mortality and ICU admission rates AUC was calculated for both CURB-65 and B/A ratios. Cox regression method was also used to investigate $\mathrm{B} / \mathrm{A}$ values with the time to mortality. Appropriate Ethical Clearance was taken from the IEC of the respective institute.

\section{Results}

A total of 112 patients were enrolled in our study out of whom $40(35.7 \%)$ required ICU care and $22(19.6 \%)$ succumbed to their disease at 30 days following admission. Univariate analysis of the various risk factors predicting ICU admission and mortality were carried out and the results are tabulated in Table 1 and Table 2. Out of the 112 patients, 15 (13.4\%) patients had comorbidities, the commonest being cardiac ailments $(9.7 \%)$, followed by diabetes $(8 \%)$, COPD $(7.1 \%)$ and chronic kidney disease $(3.6 \%)$. On univariate analysis, COPD emerged as a risk factor for mortality on univariate analysis while both COPD and cardiac ailments came out to be risk factors for ICU admission. Out of the eight patients with COPD, sputum culture yielded significant

ROC curve of B/A ratio as a predictor of Mortality

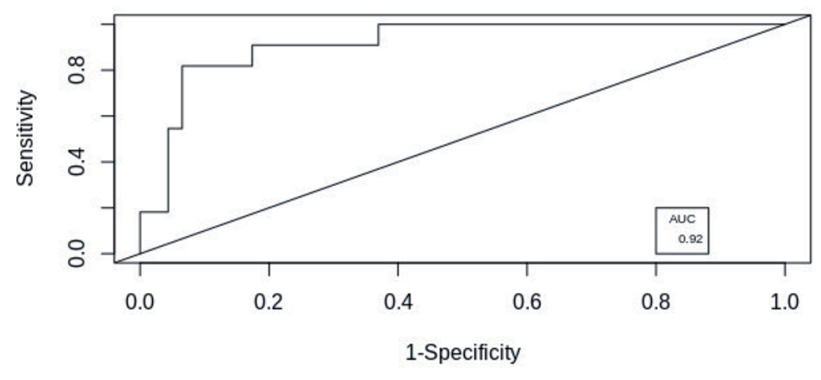

Figure 3. ROC curve of B/A ratio as a predictor of mortality.

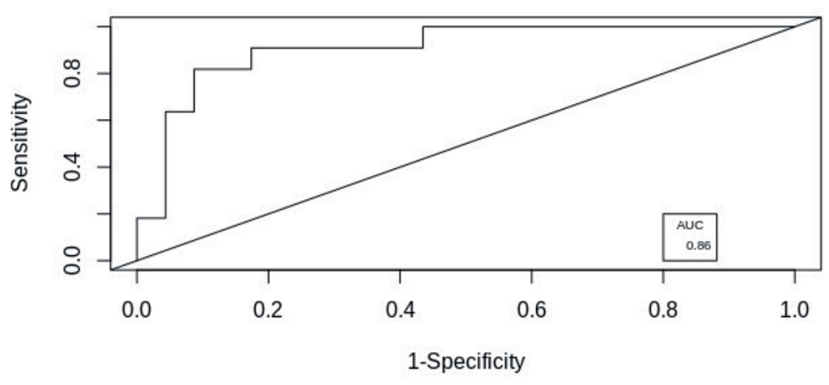

Figure 4. ROC curve of $\mathrm{B} / \mathrm{A}$ ratio as a predictor of ICU admission. 
growth in $5(62.5 \%), 3(60 \%)$ out of which had a growth of Pseudomonas spp, while the rest $2(40 \%)$ had a growth of Klebsiella spp.

Multiple logistic regression was then carried out on the risk factors that came out to be statistically significant in the univariate analysis. In the logistic regression age, CURB-65 score, B/A ratio and procalcitonin levels at admission came out to be the independent risk factors that predict risk of ICU admission and mortality within 30 days of admission (Table 3). ROC curves were carried out for B/A and CURB-65 and further AUC were calculated. AUC

Table 1. Risk factors between patients who survived and expired.

\begin{tabular}{|c|c|c|c|}
\hline Characteristics (mean \pm SD) & Survived patients ( $n=90$ ) & Expired patients ( $\mathrm{n}=22)$ & p-value \\
\hline Total number (112) & $90(80.35 \%)$ & $22(19.6 \%)$ & \\
\hline Age (years) & $52.8 \pm 16.5$ & $71.7 \pm 8.3$ & 0.0006 \\
\hline Comorbidities present (\%) & $5.5 \%(5 / 90)$ & $45.5 \%(10 / 22)$ & $<0.0001$ \\
\hline COPD, n (\%) & $1 / 5(20 \%)$ & $7 / 10(70 \%)$ & 0.034 \\
\hline ILD, n (\%) & $0 / 5$ & $1 / 10(10 \%)$ & 0.46 \\
\hline Post tubercular sequelae, $\mathrm{n}(\%)$ & $1 / 5(20 \%)$ & $0 / 10$ & 0.15 \\
\hline Cardiovascular ailments, n (\%) & $3 / 5(60 \%)$ & $8 / 10(80 \%)$ & 0.2 \\
\hline Chronic kidney disease, n (\%) & $1 / 5(20 \%)$ & $3 / 10(30 \%)$ & 0.34 \\
\hline Diabetes mellitus, n (\%) & $2 / 5(40 \%)$ & $7 / 10(70 \%)$ & 0.13 \\
\hline Procalcitonin(ng/ml) & $1.5 \pm 2.3$ & $13.4 \pm 3.4$ & 0.0001 \\
\hline Total Leukocyte counts (x $\left.10^{3} / \mu \mathrm{L}\right)$ & $16.1 \pm 10.6$ & $13.1 \pm 6.6$ & 0.38 \\
\hline Blood Urea Nitrogen (mg/dl) & $18.2 \pm 9.5$ & $37.6 \pm 22.9$ & $<0.0001$ \\
\hline Albumin (gm/dl) & $3.0 \pm 0.5$ & $2.2 \pm 0.25$ & $<0.0001$ \\
\hline BUN/Albumin ratio & $6.3 \pm 3.6$ & $16.4 \pm 7.7$ & $<0.0001$ \\
\hline $\mathrm{PaO2}$ at admission (mmHg) & $66.8 \pm 11.35$ & $57.8 \pm 10.6$ & 0.02 \\
\hline CURB-65 score & $1.36 \pm 0.79$ & $2.72 \pm 0.44$ & $<0.0001$ \\
\hline
\end{tabular}

Students' $t$-test for means or proportions used as appropriate.

Table 2. Risk factors between patients requiring and not requiring ICU admission.

\begin{tabular}{|c|c|c|c|}
\hline Characteristics (mean \pm SD) & ICU admission required & ICU admission not required & p-value \\
\hline Total number (112) & $40(35.7 \%)$ & $72(64.28 \%)$ & \\
\hline Age (years) & $65.1 \pm 12.6$ & $50.9 \pm 16.75$ & 0.0009 \\
\hline Comorbidities present (\%) & $25 \%(10 / 40)$ & $6.9 \%(5 / 72)$ & 0.004 \\
\hline COPD, n (\%) & $7 / 10(70 \%)$ & $1 / 5(20 \%)$ & 0.034 \\
\hline ILD, n (\%) & $1 / 10(10 \%)$ & $0 / 5$ & 0.46 \\
\hline Post tubercular sequelae, n (\%) & $0 / 10$ & $1 / 5(20 \%)$ & 0.15 \\
\hline Cardiovascular ailments, n (\%) & $9 / 10(90 \%)$ & $2 / 5(40 \%)$ & 0.02 \\
\hline Chronic kidney disease, n (\%) & $2 / 10(20 \%)$ & $2 / 5(40 \%)$ & 0.21 \\
\hline Diabetes mellitus, n (\%) & $6 / 10(60 \%)$ & $3 / 5(60 \%)$ & 0.5 \\
\hline Procalcitonin(ng/ml) & $8.4 \pm 12.47$ & $1.1 \pm 1.3$ & 0.001 \\
\hline Total leukocyte counts (x $10^{3} \mu \mathrm{l}$ ) & $15.97 \pm 9.74$ & $15.4 \pm 10.16$ & 0.57 \\
\hline Blood urea nitrogen (mg/dl) & $29.6 \pm 17.62$ & $17.05 \pm 9.09$ & 0.0009 \\
\hline Albumin (gm/dl) & $2.5 \pm 0.5$ & $3.11 \pm 0.45$ & 0.0001 \\
\hline BUN/albumin ratio & $12.5 \pm 6.63$ & $6.09 \pm 3.53$ & $<0.0001$ \\
\hline $\mathrm{PaO} 2$ at admission $(\mathrm{mmHg})$ & $61.92 \pm 12.5$ & $67.5 \pm 10.27$ & 0.078 \\
\hline CURB-65 score & $2.45 \pm 0.59$ & $1.13 \pm 0.62$ & $<0.0001$ \\
\hline
\end{tabular}

Students' $t$-test for means and proportions used as applicable.

Table 3. Risk factors and their association with mortality and ICU admission (multiple logistic regression).

\begin{tabular}{|c|c|c|c|c|}
\hline \multirow{2}{*}{ Risk factors } & \multirow{2}{*}{\multicolumn{2}{|c|}{ p-value (multiple logistic regression) }} & \multicolumn{2}{|c|}{ Odds ratio $(95 \% \mathrm{CI})$} \\
\hline & Mortality & & Mortality & ICU admission \\
\hline Age (years) & 0.03 & 0.01 & $1.3(1.08-1.52)$ & $2.1(1.5-2.7)$ \\
\hline BUN/albumin ratio & $7 * 10-6$ & 0.0013 & $67.8(49.2-95.4)$ & $11.2(8.4-14)$ \\
\hline Procalcitonin (ng/ml) & 0.026 & 0.048 & $1.4(1.10-1.3)$ & $1.02(1.01-1.03)$ \\
\hline CURB-65 score & 0.012 & 0.007 & $2.08(1.5-2.56)$ & $8.9(6.3-11.5)$ \\
\hline
\end{tabular}


for $\mathrm{B} / \mathrm{A}$ for ICU admission and mortality were 0.86 and 0.92 , respectively. AUC for CURB-65 score and B/A for ICU admission and mortality were 0.83 and 0.86 , respectively. Cut off values of $\mathrm{B} / \mathrm{A}$ ratio for predicting mortality came out to be $10.2 \mathrm{mg} / \mathrm{g}$ with a sensitivity of 0.7 , specificity of 0.92 , positive predictive value of $82.3 \%$ and a negative predictive value of $91.89 \%$. The optimal cut off value of B/A for ICU admission came out to be $9.84 \mathrm{mg} / \mathrm{g}$ with sensitivity of 0.68 , specificity of 0.88 , positive predictive value of $80 \%$ and a negative predictive value of $89 \%$. Out of the 112 enrolled patients, bacteriological confirmation was done in 81 $(72.32 \%)$ patients. Causative pathogens are enumerated in Table 4, the commonest pathogens isolated being Streptococcus pneumoniae $(25 \%)$ and Pseudomonas aeruginosa (16.9\%). Cox regression was done which revealed that B/A levels were significantly associated with time to mortality within 30 days of admission $(p=0.002$ with concordance level 0.94).

\section{Discussion}

Blood urea nitrogen (BUN) levels are determined by the complex balance between urea production, urea metabolism and urea excretion. The serum level of BUN is determined by many factors which can be renal or non-renal. These factors include glomerular filtration, tubular reabsorption of urea, dietary protein intake, parenteral hyperalimentation therapy, catabolism of endogenous proteins, exogenous glucocorticoid dependent catabolism, volume status and upper gastrointestinal bleeding [10]. Due to this complex interplay of modulatory factors, BUN is generally used as a surrogate marker of systemic illness rather than a specific marker of renal dysfunction. In patients with CAP there is an infective focus which may lead to sepsis and systemic inflammatory response, and this leads to worse clinical outcomes. The high rates of mortality in this subgroup of patients may also be due to the neurohormonal response to arterial underfilling due to systemic vasodilatation following septicaemia. The neurohormonal response includes activation of the renin-angiotensin cycle and production of AVP [11]. High plasma AVP concentrations can result in increased urea reabsorption in the collecting duct, resulting in an increased BUN [12]. Angiotensin and adrenergic stimulation increase proximal tubular sodium and water reabsorption, decreasing distal fluid delivery which increases flow-dependent urea reabsorption [13]. In the study by Farr et al., in which 245 patients with CAP were studied, BUN was shown to be an independent risk factor for mortality $(p<0.0001)$ [14]. In the study by Raz et al., in which 320 patients were enrolled, mortality was found in $14.4 \%$ patients within 1 month. BUN $>30 \mathrm{mg} / \mathrm{dl}$ was found to be found to be an independent

Table 4. Causative pathogens.

\begin{tabular}{lc} 
Microorganisms & n (\%) \\
Streptococcus pneumoniae & $23(20.5 \%)$ \\
Pseudomonas aeruginosa & $19(16.9 \%)$ \\
\hline Klebsiella pneumoniae & $10(13.4 \%)$ \\
Staphylococcus aureus & $9(8.03 \%)$ \\
\hline Haemophilus influenzae & $5(4.4 \%)$ \\
Escherichia coli & $4(3.6 \%)$ \\
\hline Moraxella catarrhalis & $1(0.9 \%)$ \\
H1N1 & $10(12.3 \%)$ \\
\hline
\end{tabular}

risk factor for mortality in this study too with Odds ratio of 7.8 (3.7-16.4) [15].

Low albumin level has been shown to predict poor outcome in many patients including CAP. The quantity of albumin production is markedly decreased in the acute phase of inflammation. Many of the patients of pneumonia are infected with gram negative bacteria. These bacteria promote the release of cytokines, interleukins and chemokines as mediators of inflammation. These mediators increase the membrane permeability and lead to escape of albumin from the capillary vessels. In the study by Lee et al, in which 424 patients were enrolled, serum albumin emerged as an independent risk factor for 28-day mortality with a hazard ratio of 0.37 (0.19- 0.73) [7].

In recent years, studies have shown $\mathrm{B} / \mathrm{A}$ ratio to be an important marker to predict short term mortality and morbidity in patients with CAP. In the study by Jyothi et al., the optimal level of BUN/Albumin to establish the necessity for ICU management was $\geq 12.94 \mathrm{mg} / \mathrm{g}$. The sensitivity $91.30 \%$ and specificity being $65.79 \%$ [16]. In the study by Ugajin et al. [8], B/A ratio had an AUC of 0.83 (95\% CI $0.73-0.94)$ for mortality and an AUC of 0.86 (95\% CI 0.79-0.94) for ICU admission. The optimal cut-off value of the B/A ratio for predicting mortality was $12.44 \mathrm{mg} / \mathrm{g}$ and ICU admission was $9.85 \mathrm{mg} / \mathrm{g}$. In comparison, the AUC of CURB -65 with respect to ICU admission was 0.81 (95\% CI 0.71-0.91) and for mortality was 0.84 (95\% CI $0.77-0.91)$ [8]. In our study too, B/A outperformed CURB 65 score as a better differentiator for predicting ICU admission (0.86 vs 0.83$)$ and mortality (0.92 vs 0.86$)$.

Patients with structural lung diseases, especially COPD are a higher risk of developing CAP and suffering worse clinical outcomes, including need for ICU admission and 30-day mortality $[17,18]$. In our study too, it was found to be a significant risk factor for ICU admission and mortality. This significance disappeared on multiple logistic regression, maybe due to the fact that only eight of the 112 enrolled patients had a spirometric diagnosis of COPD at time of admission. Prevalence of COPD in India is vastly underestimated, determined to be $4.2 \%$, in comparison to USA where the prevalence is around $10-21 \%$ [19]. This is mostly due to lack of information and less availability of diagnostic centres. Also, we believe that along with presence of COPD, the severity of obstruction would be a strong risk factor in predicting clinical outcomes which was not included in our study.

$\mathrm{B} / \mathrm{A}$ ratio has the benefit of being easily calculated and not dependent on operator capacity to correctly gauge the level of confusion in a patient as required in the CURB-65 score. We believe that instead of cumbersome scoring systems like PSI (Pneumonia Severity Index) or APACHE-II, B/A ratio has the potential to become an important surrogate marker for complications in patients with CAP. It will help the treating clinician to streamline patients who are at a higher risk and start intensive care at an earlier stage of the disease process. This would lead to better utilisation of resources and better clinical outcomes in patients.

We acknowledge a few limitations of our study. Our sample size was small, consisting of only 112 patients. Hence we believe to correctly calculate the optimal cut off value of B/A ratio, larger studies should be conducted. In patients with renal diseases, the values of B/A could be falsely elevated. However, role of B/A ratio as a prognosticator in patients with pre-existing renal disease could not be properly studied as patients with CKD formed a small proportion of the patients. The role of $\mathrm{B} / \mathrm{A}$ ratio at the time of admission was used in our study but we believe that serial $\mathrm{B} / \mathrm{A}$ ratios over a period would better reflect the trend of the disease. Further studies to look at persistently raised $\mathrm{B} / \mathrm{A}$ ratios in predicting mortality and morbidity would better help us to understand its role in CAP. 


\section{References}

1. GBD 2016 Lower Respiratory Infections Collaborators. Estimates of the global, regional, and national morbidity, mortality, and aetiologies of lower respiratory infections in 195 countries, 1990-2016: A systematic analysis for the Global Burden of Disease Study 2016. Lancet Infect Dis 2018;18:1191-210.

2. Ramirez JA, Wiemken TL, Peyrani P, et al. Adults hospitalized with pneumonia in the United States: Incidence, epidemiology, and mortality. Clin Infect Dis 2017;65:1806-12.

3. Lim WS, van der Eerden MM, Laing R, et al. Defining community acquired pneumonia severity on presentation to hospital: an international derivation and validation study. Thorax 2003;58:377-82.

4. Rudolph JL, Zanin NM, Jones RN, et al. Hospitalization in community-dwelling persons with Alzheimer's disease: frequency and causes. J Am Geriatr Soc 2010;58:1542-8.

5. Shah BA, Ahmed W, Dhobi GN, et al. Validity of pneumonia severity index and CURB- 65 severity scoring systems in community acquired pneumonia in an Indian setting. Indian $\mathrm{J}$ Chest Dis Allied Sci 2010;52:9-17.

6. Potgieter PD, Hammond JM. The intensive care management, mortality, and prognostic indicators in severe communityacquired pneumococcal pneumonia. Intensive Care Med 1996; 22:1301-6.

7. Lee JH, Kim J, Kim K, et al. Albumin and C-reactive protein have prognostic significance in patients with communityacquired pneumonia. J Crit Care 2011;26:287-94.

8. Ugajin M, Yamaki K, Iwamura N, et al. Blood urea nitrogen to serum albumin ratio independently predicts mortality and severity of community-acquired pneumonia. Int J Gen Med 2012;5:583-9.

9. Akpinar EE, Hoşgün D, Doğanay B, Gülhan M. The role of albumin level and blood urea nitrogen/albumin ratio in prediction of prognosis of community acquired pneumonia. J Pulm Respir Med 2013;3:5.

10. Beier K, Eppanapally S, Bazick HS, et al. Elevation of blood urea nitrogen is predictive of long-term mortality in critically ill patients independent of "normal" creatinine. Crit Care Med 2011;39:305-13.

11. Schrier RW. Body fluid volume regulation in health and disease: a unifying hypothesis. Ann Intern Med 1990;113:155-9.

12. Conte G, Dal Canton A, Terribile M, et al. Renal handling of urea in subjects with persistent azotemia and normal renal function. Kidney Int 1987;32:721-7.

13. Schrier RW. Blood urea nitrogen and serum creatinine: not married in heart failure. Circ Heart Fail 2008;1:2-5.

14. Farr BM, Sloman AJ, Fisch MJ. Predicting death in patients hospitalized for community- acquired pneumonia. Ann Intern Med 1991;115:428-36.

15. Raz R, Dyachenko P, Levy Y, et al. A predictive model for the management of community- acquired pneumonia. Infection 2003;31:3-8.

16. Jyothi R, Basavaraj B, Gurupadappa K. The prognostic implication of serum albumin and BUN/Albumin ratio in assessing severity and mortality in community acquired pneumonia (CAP). Int J Clin Biochem Res 2019;6:79-81.

17. Dai RX, Kong QH, Mao B, et al. The mortality risk factor of community acquired pneumonia patients with chronic obstructive pulmonary disease: a retrospective cohort study. BMC Pulm Med 2018;18:12.

18. Restrepo MI, Mortensen EM, Pugh JA, Anzueto A. COPD is associated with increased mortality in patients with community-acquired pneumonia. Eur Respir J 2006;28:346-51.

19. India State-Level Disease Burden Initiative CRD Collaborators. The burden of chronic respiratory diseases and their heterogeneity across the states of India: the Global Burden of Disease Study 1990-2016. Lancet Glob Health 2018;6:e1363-74. 